\section{Reparación de hernia umbilical, análisis general del tratamiento quirúrgico}

Umbilical hernia repair, overview of surgical treatment

Tatiana Giomara Gómez García

Médico general. Centro de Salud Tipo

A Distrito 06d02,

tatianag.gomezgarcia@gmail.com,

https://orcid.org/0000-0001-9213-

4038, Guayaquil, Ecuador

Edison Javier Siguencia Matute

Médico general. Hospital Clínica

Kennedy,

javiersiguencia2017@gmail.com,

https://orcid.org/0000-0002-8997-

2361, Guayaquil, Ecuador

Javier Stalin Villagómez Toral

Médico general. Centro médico

Medilink Sur,

javime8590@outlook.com,

https://orcid.org/0000-0002-8997-

0657, Guayaquil, Ecuador

Dayana Lissette Miranda Espín

Médico general. Centro de Salud

Sociovivienda 1

dra.dayimiranda@gmail.com,

https://orcid.org/0000-0001-5073-

3892, Guayaquil, Ecuador

http://www.jah-

journal.com/index.php/jah

Journal of American health

Julio - Diciembre vol. 3. Num. 2 - 2020

Esta obra está bajo una Licencia

Creative Commons

Atribución-NoComercial-

CompartirIgual 4.0 Internacional.

RECIBIDO: 28 DE ABRIL 2019

ACEPTADO: 29 DE AGOSTO 2019

PUBLICADO: 2 DE JULIO 2020

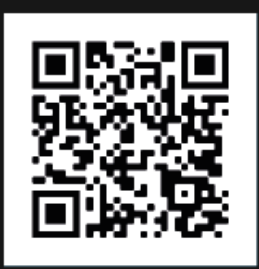

Scan this QR

code with your

smart phone or

mobile device to

read more papers

\section{RESUMEN}

Actualmente no existe consenso sobre la mejor técnica para la reparación de la hernia umbilical en adultos. El papel de la hernioplastia laparoscópica de la hernia umbilical sigue siendo controvertido. Se utilizó un repositorio digital como Pubmed para la búsqueda avanzada de la bibliografía sobre el tema, usando las palabras clave específicas. Se analizaron artículos con menos de diez años de publicación que resultaron de la búsqueda avanzada de la información. Conclusión: Esta revisión de la literatura aclara algunos aspectos del tratamiento quirúrgico de la hernia umbilical: el tipo de procedimiento debe estar determinado por los requisitos de la situación clínica. La reparación laparoscópica se asocia con un menor riesgo de infección, dehiscencia de la herida, recurrencia, estadía más corta pero mayor tiempo operatorio. Las técnicas abiertas se usan con mayor frecuencia debido al pequeño tamaño de estas hernias. Las reparaciones con malla disminuyen las tasas de recurrencia. Las reparaciones laparoscópicas generalmente son adecuadas para pacientes obesos 0 pacientes con alto riesgo de complicaciones de la herida. Se necesitan más estudios con tecnología robótica para aclarar su beneficio.

PALABRAS CLAVE: hernia umbilical, tratamiento, malla, laparoscopía.

\section{ABSTRACT}

There is currently no consensus on the best technique for repairing umbilical hernia in adults. The role of laparoscopic hernia repair of umbilical hernia remains controversial. A digital repository such as Pubmed was used for the advanced search 
of the bibliography on the topic, using specific keywords. Articles were analyzed with less than three years of publication that resulted from the advanced search for information. Conclusion: This review of the literature clarifies some aspects of the surgical treatment of umbilical hernia: the type of procedure must be determined by the requirements of the clinical situation. The laparoscopic repair is associated with a lower risk of infection, dehiscence of the inheritance, recurrence, the stage was shorter but the higher operating time. Open techniques are used with greater frequency due to the small size of these hernias.
Repairs with the mall will decrease the recurrence rates. Laparoscopic repairs are generally suitable for obese patients or patients with a high risk of complications from the disease. More studies with robotic technology are needed to clarify your benefit.

KEYWORDS: umbilical hernia, treatment, mesh, laparoscopy.

\section{INTRODUCCIÓN}

Las hernias umbilicales son algunas de las hernias más comunes encontradas por los cirujanos; representan el $10-12 \%$ de las hernias de la pared abdominal (1). Aproximadamente 175,000 hernias umbilicales son reparadas quirúrgicamente anualmente en los Estados Unidos (2). Aunque las hernias umbilicales a menudo se consideran hernias simples, pueden ser complejas $y$, si no se manejan adecuadamente, pueden ser irritantes para pacientes y cirujanos. Una verdadera hernia umbilical se clasifica como una hernia primaria; sin embargo, debido a que el ombligo a menudo se usa para el acceso laparoscópico, pueden ocurrir hernias incisionales en el ombligo y los cirujanos deben estar al tanto de las distinciones entre las dos.

Dentro de las opciones de tratamiento de la hernia umbilical, se puede realizar una reparación de sutura primaria simple para defectos pequeños. Las hernias umbilicales se han reparado históricamente sin malla. Mayo fue el primero en describir la técnica de superposición de la fascia de la pared abdominal y siguió siendo la técnica quirúrgica más reconocida durante mucho tiempo, hasta que se demostró que la reparación de Mayo está asociada con altas tasas de recurrencia de hasta $28 \%(1,2,3)$. Las tasas de recurrencia relativamente altas asociadas con las técnicas de sutura primaria aumentaron la popularidad de la reparación con mallas. La introducción de la reparación con malla ha mejorado las tasas de recurrencia después de las reparaciones de hernia umbilical.

La reparación laparoscópica de hernia umbilical se describió en la década de 1990. El uso de la reparación laparoscópica de hernias ventrales ha aumentado en los últimos años. La literatura sugiere que el abordaje laparoscópico tiene las ventajas de tasas de recurrencia reducidas, dolor postoperatorio menor, estadía hospitalaria más corta y una tasa de morbilidad 
disminuida, aunque está potencialmente asociado con un tiempo operatorio relativamente prolongado.

Varias cosas hacen que las hernias umbilicales sean un desafío, incluida la heterogeneidad de la presentación, múltiples opciones de reparación, y potencial de complicaciones, incluyendo infección y recurrencia. Actualmente continúa el debate sobre las indicaciones para el uso de mallas para hernias umbilicales, técnicas óptimas para la reparación y el papel de la tecnología robótica para la reparación. Aunque las hernias umbilicales son algunas de las hernias reparadas con mayor frecuencia en el mundo. todavía hay muchas preguntas sin respuesta relacionadas con su reparación, y la literatura sobre el tema es escasa, con pocos estudios bien hechos. Por tal motivo, la mejor técnica quirúrgica para la reparación de hernias umbilicales sigue siendo controvertida.

\section{MATERIALES Y MÉTODOS}

Se realizó una búsqueda de literatura médica para conocer las características generales de las técnicas de reparación de hernia umbilical en bases de datos como PubMed, Elsevier y Scielo. La búsqueda incluyó articulos desde enero del 2010 hasta abril del 2020. Se utilizaron estrategias rastreo de información científica con los siguientes términos del vocabulario MeSH: "umbilical hernia repair" OR "umbilical hernia repair" OR "Suture Techniques" OR "adverse effects" OR "outcome Assessment" AND "Postoperative Complications"; y del vocabulario DeCS: hernia umbilical, tratamiento, malla, laparoscopía y sutura primaria. Se aplicaron los siguientes criterios de inclusión: artículos de revisión narrativa o sistemática publicados en los últimos 11 años y realizados en seres humanos. No se realizó descarte según el idioma. El criterio de exclusión fue artículos cuyo contenido era sobre la fisiopatología y epidemiología. Para la lectura crítica en texto completo se escogieron 10 artículos y se adicionaron 5 artículos más procedentes de las citaciones de los documentos iniciales, los cuales se consideraron relevantes para la revisión del tema.

\section{RESULTADOS}

Una hernia se define como una protuberancia de una estructura o parte de la estructura a través de los tejidos que normalmente la contienen. En general, una hernia es congénita 0 adquirida. Existen múltiples teorías sobre cómo y por qué las hernias se desarrollan con el tiempo, y lo más probable es que cada hernia tenga una etiología multifactorial (1). Además, actualmente no es posible en la mayoría de los casos determinar qué factores son más importantes para un paciente determinado en un momento dado. Los factores etiológicos son diferentes entre pacientes e incluso diferentes para un solo paciente a lo largo del tiempo. Por ejemplo, un paciente puede desarrollar una hernia traumática de un golpe directo a la pared abdominal en un punto en el tiempo, pero desarrolla una hernia incisional en otro punto debido a una infección de la herida postoperatoria y/o técnica de cierre (2). Incluso conocer las 
posibles etiologías para esos dos escenarios clínicos nos deja en la oscuridad sobre un trastorno de colágeno no diagnosticado que de otra manera no tiene manifestaciones clínicas

Para este artículo, definimos las hernias adquiridas en la línea media como todas aquellas diagnosticadas durante la edad adulta, reconociendo el hecho de que algunas de ellas habrán estado presentes al nacer y que el paciente y/o la comunidad médica pasaron desapercibidas durante años. El embarazo, el aumento de peso, obesidad, tumores intraabdominales y la ascitis pueden aumentar la presión dentro del abdomen, causando un aumento en el tamaño de un defecto en la pared abdominal umbilical o epigástrica. La otra consecuencia de este aumento de la presión puede ser una mayor probabilidad de encarcelamiento o estrangulamiento de la grasa preperitoneal (ligamentos falciformes o umbilicales), epiplón o intestino (3).

Los pacientes a veces se quejan de sentir que una hernia "sale" repentinamente, especialmente aquellos que participan en levantar objetos pesados en el trabajo, levantar pesas, toser o cualquier otro evento que cause un esfuerzo significativo del abdomen. En el pasado, se pensaba que el aumento extremo transitorio de la presión intraabdominal causaba la hernia, pero estudios más recientes han demostrado que probablemente ese no sea el caso (2). Se han realizado investigaciones que sugieren que los pacientes con hernias tienen menos colágeno tipo I y más elastina en la línea alba que los pacientes sin hernias. Este tipo de trastorno del tejido conectivo no es clínicamente aparente, pero probablemente predispone a ciertas personas a desarrollar una hernia. Por supuesto, las fuentes más obvias de trauma, como la fuerza contundente de un choque automovilístico o un fuerte golpe en el abdomen, también pueden causar una hernia.

Una hernia umbilical es una hernia ventral ubicada en o cerca del ombligo. A veces se les conoce como hernia "periumbilical", porque no siempre se localizan inmediatamente en la base del ombligo. Según la anatomía de Gray, el área que rodea la cicatriz que llena el defecto umbilical es más débil que la cicatriz misma, lo que hace que todas las hernias ventrales en el ombligo sean "peri-umbilicales" (2,3). Más recientemente, en 2011, Eltair et al intentaron caracterizar la pared abdominal en el anillo umbilical para determinar qué puede predisponer a los pacientes a desarrollar hernias umbilicales. Combinaron 2 sistemas de clasificación de anillo umbilical previos en 5 tipos en un intento de definir mejor las características morfológicas en el anillo umbilical y su relación con los ligamentos umbilicales y falciformes adyacentes (4). Parece que el ligamento falciforme (o redondo) puede funcionar para proteger contra la hernia cuando cruza y cubre el anillo umbilical, insertando a lo largo del borde inferior del anillo.

\section{TÉCNICAS ABIERTAS Y USO DE MALLA}

Una decisión importante que enfrentan los cirujanos cuando planifican una reparación abierta de hernia umbilical se relaciona con el uso de mallas. El uso de malla y las indicaciones precisas para la reparación de malla y sin malla se debaten sin un consenso claro. Cuatro ensayos aleatorios prospectivos han evaluado el uso de malla para hernias umbilicales; tres de cuatro 
informaron tasas de recurrencia más bajas con el uso de mallas, con el mayor beneficio en pacientes con cirrosis y pacientes sometidos a reparación emergente de hernias encarceladas $(4,5,6)$. Un estudio basado en la población danesa informó hallazgos similares. Las tasas de recurrencia fueron menores en pacientes sometidos a reparación de malla en comparación con la reparación sin malla en 4786 pacientes sometidos a reparación abierta electiva de hernias umbilicales o epigástricas pequeñas $(2 \mathrm{~cm})(7)$.

Reparación primaria

La reparación de Mayo, descrita por primera vez en 1901, fue una reparación de sutura primaria de hernias umbilicales descrita como un cierre fascial "chaleco sobre pantalones" en una orientación transversal utilizando 2 filas de suturas colchonero horizontales (3). Aunque esta técnica fue popular durante muchos años y se usa ocasionalmente en la actualidad, las altas tasas de recurrencia a largo plazo han limitado su uso con la mayoría de los cirujanos que usan un cierre primario simple, interrumpido o figura de 8 de manera horizontal.

Las técnicas de reparación primaria abierta varían. Sin embargo, en general, los pacientes deben prepararse de manera similar a otras operaciones abdominales con anestesia general y antibióticos perioperatorios (1). La mayoría de las hernias umbilicales pueden abordarse a través de una incisión infraumbilical curvilínea de $3 \mathrm{~cm}$ a $4 \mathrm{~cm}$ en el pliegue infraumbilical. La disección roma se inicia a ambos lados del ombligo para rodear el tallo umbilical y el saco herniario. Por lo general, se coloca una pinza hemostática por encima y alrededor del tallo umbilical, y el saco herniario se diseca del tallo (2). Es importante evitar abotonar la piel en esta coyuntura de operación. Esto se puede evitar colocando un dedo o un instrumento romo en el ombligo para ayudar a identificar la unión. Una vez que el saco de la hernia se diseca libremente, se puede abrir para identificar el contenido. Cuando hay grasa preperitoneal $u$ epiplón presente, puede reducirse nuevamente en la cavidad abdominal o simplemente extirparse a nivel fascial (3). En este punto, los bordes fasciales deben estar claramente identificados.

La mayoría de los cirujanos disecan aproximadamente $1 \mathrm{~cm}$ a $2 \mathrm{~cm}$ de tejido subcutáneo de la fascia para garantizar una identificación clara para la sutura. Debido a que no hay estudios relacionados con la técnica de sutura para la reparación de la hernia umbilical, esto debe dejarse a discreción del cirujano y generalmente implica suturas interrumpidas o en forma de ocho $(1,2,3)$. Aunque no hay evidencia convincente sobre el tipo de material de sutura que debe usarse, según algunos datos de un estudio danés, parece haber una tasa de recurrencia más alta usando suturas absorbibles en comparación con las suturas no absorbibles, por lo que esto debe considerarse al reparar hernias umbilicales. Después de cerrar la fascia, se irriga la herida y se sutura el tallo umbilical a la fascia anterior para restaurar la forma umbilical normal. La piel se puede cerrar con una sutura absorbible y apósito aplicado. Cuando la piel umbilical se ha estirado desde la hernia, se puede aplicar un apósito a presión para reducir potencialmente la formación de seroma. 
Reparaciones basadas en mallas

Como se discutió anteriormente, la decisión con respecto al uso de la malla está relacionada con muchos factores del paciente y características de la hernia. En algunos casos, esta decisión se toma en el momento de la cirugía después de examinar el defecto de la hernia y la integridad de la fascia (6). Cuando se toma la decisión de usar la malla, los riesgos y beneficios propuestos deberían haberse discutido con el paciente antes de la operación. Después de tomar la decisión de usar la malla, el cirujano debe elegir el mejor tipo de malla y ubicación de colocación. En general, la mayoría de las reparaciones electivas de hernia umbilical son limpias, por lo que generalmente se recomienda una malla sintética permanente. Existen pocos datos relacionados con el uso de mallas biológicas o bioabsorbibles debido a las complicaciones infecciosas de la herida y la malla bastante bajas asociadas con las reparaciones de hernia umbilical basadas en la malla. Por lo tanto, es probable que estas costosas tecnologías de malla tengan pocos beneficios y solo se usen para indicaciones específicas.

La elección de la malla debe basarse en la técnica de reparación elegida. Al igual que en otras reparaciones de hernia ventral, la malla se puede colocar como una subcapa (una posición intraabdominal, preperitoneal o retrorectus), una incrustación (tapón de malla suturado a los bordes fasciales) o una incrustación (malla colocada sobre la fascia principalmente cerrada). Al igual que con las reparaciones de malla para hernias ventrales en general, no hay consenso sobre la ubicación ideal para la colocación de malla, y esto debe basarse en el escenario clínico $(4,6)$. El conocimiento de las posibles ventajas y desventajas de los diferentes productos de malla y ubicaciones de malla es esencial. En general, las técnicas de incrustación tienen tasas de recurrencia más altas y, por lo tanto, no deben usarse de manera rutinaria.

Técnicas laparoscópicas

En la actualidad, el uso de la laparoscopía para la reparación de la hernia umbilical es bastante bajo. Los estudios han indicado que la laparoscopia se usa solo en una cuarta parte de los casos. La mayor parte de las guías y protocolos actuales informan una posible disminución en la morbilidad total y de la herida asociada con la reparación laparoscópica en comparación con la reparación abierta, electiva y primaria de hernias umbilicales (2). Esto se produjo a expensas del tiempo operatorio prolongado y la estadía en el hospital, con un aumento de las complicaciones respiratorias y cardíacas. Después de controlar los factores del paciente, incluido el índice de masa corporal, el género, la clase de la Sociedad Estadounidense de Anestesiólogos (ASA) y la enfermedad pulmonar obstructiva crónica, la razón de probabilidades de complicaciones generales favoreció el abordaje laparoscópico, que fue impulsado por la reducción de las complicaciones de la lesión (8).

La reparación laparoscópica de la hernia está indicada por varias razones, incluida la obesidad, los defectos fasciales grandes y la reparación previa de la hernia. El enfoque laparoscópico le permite al cirujano definir inequívocamente los márgenes del defecto de la hernia e identificar defectos fasciales adicionales o hernias ocultas. Algunas hernias incluyen defectos faciales 
pequeños adyacentes al defecto grande $y$, cuando se omiten, esto puede aumentar las tasas de recurrencias $(8,9)$. El enfoque laparoscópico también permite una cobertura de malla más amplia, porque la malla se coloca en la posición intraperitoneal. Esta técnica ha resultado en una disminución del dolor postoperatorio, un retorno más corto a la actividad normal y menores tasas de recurrencia (10).

\section{DISCUSIÓN}

Pocos estudios están disponibles para guiar al cirujano sobre el tratamiento quirúrgico de las hernias umbilicales. En cirugía general, se recomienda el tratamiento para pacientes sintomáticos. Dos estudios evaluaron las estrategias de cirugía y espera vigilante para hernias umbilicales y tienen conclusiones diferentes. Strosberg et al (3) evaluaron una gran base de datos de 279 pacientes con hernias umbilicales. Informaron un costo financiero más alto en el grupo quirúrgico, pero significativamente más días de la atención médica y más días estimados de trabajo perdidos en el grupo sin cirugía y recomendaron una intervención quirúrgica temprana para reducir potencialmente el costo y la utilización de recursos. Kokotovic et al (11), evaluaron 569 pacientes con hernias incisionales y 789 pacientes con hernias umbilicales e informaron una probabilidad del $16 \%$ de pacientes con hernias umbilicales para requerir cirugía durante 5 años y una probabilidad del $4 \%$ de requerir cirugía de emergencia. Llegaron a la conclusión de que la espera vigilante es una estrategia segura en pacientes con hernias umbilicales.

Los estudios que hacen referencia a los resultados sobre la reparación laparoscópica y abierta de la hernia umbilical. Cassie et al (8), evaluaron los resultados de las reparaciones abiertas y laparoscópicas de hernias umbilicales electivas en 14,652 pacientes. Informaron una disminución de las complicaciones de la herida asociadas con la reparación laparoscópica, pero aumentaron los tiempos de operación, la duración de la estadía y las complicaciones respiratorias y cardíacas. Este concepto de disminución de la morbilidad de la herida en la reparación de la hernia laparoscópica en comparación con abierto ha sido bien documentado; sin embargo, debe tomarse en contexto para la reparación de la hernia umbilical, porque la tasa de infección de la herida es baja.

El uso de la malla sigue siendo un tema debatido para la reparación de la hernia umbilical. Existe la suficiente evidencia, de que el uso de la malla reduce las tasas de recurrencia, pero posiblemente aumenta el riesgo de infecciones y ocurrencias en el sitio quirúrgico.

Aunque generalmente se cree que las reparaciones de hernia umbilical están asociadas con bajas tasas de recurrencia y complicaciones, hay pocos estudios que evalúen los resultados a largo plazo de este procedimiento común, y varios estudios basados en la población muestran resultados que probablemente sean más altos de lo que piensan los cirujanos (12,13). Dos estudios de Dinamarca evaluaron quejas crónicas después de la reparación de hernias umbilicales y epigástricas. Westen et al (14), encuestaron a 295 pacientes que se sometieron a 
reparación por sutura de hernias umbilicales y epigástricas con una mediana de seguimiento de 5 años e informaron que el $5.5 \%$ de los pacientes tenían quejas crónicas, como dolor o restricciones de trabajo/ocio y esto podría explicarse en parte por recurrencia de hernia. Erritzoejervild et al (15), encuestaron a 132 pacientes que fueron sometidos a sutura y reparación con malla con una mediana de seguimiento de 36 meses. Informaron que el $12 \%$ de los pacientes tenían dolor e incomodidad moderados o severos y un riesgo acumulado de recurrencia del 11.5\%. Estos 2 estudios muestran que las hernias umbilicales no son simples y los cirujanos deberían trabajar para mejorar los resultados en estos pacientes.

\section{CONCLUSIONES}

La mejor evidencia disponible sugiere que la reparación laparoscópica de hernias umbilicales o paraumbilicales puede estar asociada con un menor riesgo de infección de la herida, dehiscencia de la herida y tasa de recurrencia, estadía más corta pero mayor tiempo operatorio. Los resultados de un número limitado de ensayos clínicos aleatorizados no mostraron diferencias en las tasas de recurrencia.

Las hernias umbilicales son comunes y a menudo se consideran hernias simples; sin embargo, pueden plantear muchos desafíos al cirujano, y los resultados a largo plazo aún no son ideales. Las técnicas abiertas, laparoscópicas y posiblemente robóticas tienen un papel en la reparación de la hernia umbilical, y su uso debe estar determinado por los requisitos de la situación clínica. Las técnicas abiertas se usan con mayor frecuencia debido al pequeño tamaño de estas hernias, y las incisiones pequeñas generalmente se usan para la reparación. Se ha demostrado que las reparaciones de malla disminuyen las tasas de recurrencia de la hernia umbilical y deben usarse en la mayoría de los casos, pero pueden provocar una morbilidad de la herida ligeramente mayor.

Las reparaciones laparoscópicas generalmente son adecuadas para pacientes obesos o pacientes con alto riesgo de complicaciones de la herida. La cirugía robótica, una tecnología emergente para la reparación de hernias, puede tener algunas ventajas potenciales, pero esto debe compararse con el costo de esta tecnología. Se necesitan más estudios con tecnología robótica.

Los cirujanos deben continuar esforzándose por mejorar los resultados para los pacientes con hernias umbilicales y aclarar las indicaciones para el uso de mallas y diversas técnicas quirúrgicas

Evidencia de alto nivel para la reparación de hernia umbilical con mallas en pacientes con hernias pequeñas de 1-4 cm de diámetro. Por lo tanto, es recomendable que la reparación con malla se use para operaciones en todos los pacientes con una hernia umbilical de este tamaño. 


\section{REFERENCIAS}

1. Appleby P. Umbilical Hernia Repair: Overview of Approaches and Review of Literature. Surg Clin North Am. Vol. 98(3):561-576. doi:10.1016/j.suc.2018.02.001. 2018.

2. Hajibandeh S. Laparoscopic versus open umbilical or paraumbilical hernia repair: a systematic review and meta-analysis. Hernia. Vol. 21(6):905-916. doi:10.1007/s10029017-1683-y. 2017.

3. Chung I. Laparoscopic versus open repair for small paraumbilical hernia: A retrospective review. Asian J Endosc Surg. Vol. 12(3):306-310. doi:10.1111/ases.12644. 2019.

4. Eltair et al. Meta-analysis of laparoscopic groin hernia repair with or without mesh fixation. Int J Surg. Vol. 71:190-199. doi:10.1016/j.ijsu.2019.10.004. 2019.

5. Earle D MJ. Repair of umbilical and epigastric hernias. Surg Clin North Am. Vol. 93(5):10571089. doi:10.1016/j.suc.2013.06.017. 2013.

6. Westen et al. Chronic complaints after simple sutured repair for umbilical or epigastric hernias may be related to recurrence. Langenbecks Arch Surg. Vol. 399:65-69. 2014.

7. Erritzoe-Jervild et al. Long-term complaints after elective repair for small umbilical or epigastric hernias. Hernia. Vol. 17: 211-215. 2013.

8. Strosberg D. Umbilical hernias: the cost of waiting. Surg Endosc. Vol. 31:901-6. 16.. 2017.

9. Kokotovic et al. Watchful waiting as a treatment strategy for patients with a ventral hernia appears to be safe. Hernia. Vol. 20:281-7. 2016.

10. Christoffersen et al. Lower reoperation rate for recurrence after mesh versus sutured elective repair in small umbilical and epigastric hernias. A nationwide register study. World J Surg. Vol. 37:2548-52. 2013.

11. Ammar S. Management of complicated umbilical hernias in cirrhotic patients using permanent mesh: randomized clinical trial. Hernia. Vol. 14 (1):35-8. 2010.

12. Cassie et al. Laparoscopic versus open elective repair of primary umbilical hernias: shortterm outcomes from the American College of Surgeons National Surgery Quality Improvement Program. Surg Endosc. Vol. 28(1): 741-6. 2014.

13. Nguyen et al. Comparison of outcomes of synthetic mesh vs suture repair of elective primary ventral herniorrhaphy: a systematic review and meta-analysis. JAMA Surg. Vol. 149:415-21. 2014.

14. Berger et al. Suture versus preperitoneal polypropylene mesh for elective umbilical hernia repairs. J Surg Res. Vol. 192(1):426-31. 2014. 
15. Kaufmann et al. Mesh versus suture repair of umbilical hernia in adults: a randomised, double-blind, controlled, multicentre trial. Lancet. Vol. 391(10123):860-869. doi:10.1016/S0140-6736(18)30298-8. 2018. 